Recepción: 27 / 03 / 2018

Aceptación: 28 / 06 / 2018

\title{
Evaluación financiera en salud pública en la Amazonía Ecuatoriana
}

\section{Financial evaluation in public health in the Ecuadorian Amazon}

\section{Avaliação financeira em saúde pública na Amazônia equatoriana}

\author{
Zully S. Diaz-Alay ${ }^{\mathrm{I}}$ \\ zdiaz@upse.edu.ec \\ Jeffry J. Pavajeau-Hernández II \\ jpavajeau@upse.edu.ec \\ Sonnia A. Santos-Holguín III \\ ssantos@upse.edu.ec
}

Correspondencia: zdiaz@upse.edu.ec

I Diploma Superior en Promoción y Prevención de la Salud, Magister en Gerencia de Salud para el Desarrollo Local, Licenciada en Enfermería, Docente de la Carrera de Enfermería de la Universidad Estatal Península De Santa Elena, La Libertad, Ecuador.

II Diploma Superior en Promoción y Prevención de la Salud, Magister en Gerencia de Salud para el Desarrollo Local, Médico, Docente de la Carrera de Enfermería de la Universidad Estatal Península De Santa Elena, La Libertad, Ecuador.

III Magister en Gerencia de Innovaciones Educativas, Licenciada en Enfermería, Docente de la Carrera de Enfermería de la Universidad Estatal Península De Santa Elena, La Libertad, Ecuador. 


\title{
Resumen
}

El objetivo del presente estudio es analizar el valor de las evaluaciones financieras como herramienta para la toma de decisiones gerenciales en los establecimientos de salud en zonas de difícil acceso y cristalizar la calidad de los servicios de salud mediante la eficiencia, eficacia y efectividad. La metodología ejecutada fue mediante el estudio analítico-descriptivo de datos enfocados en ocho dispensarios rurales del seguro social campesino de la provincia de Morona Santiago durante el año 2014. Se ejecutó el costeo de los servicios de salud por centros de costos mediante el software Winsig, la facturación de las prestaciones de salud según el tarifario vigente y usando la ecuación del punto de equilibrio se determinó la realidad financiera en territorio. Se concluye que las unidades de salud rurales trabajan a pérdida trabajan, generando hasta el triple de gastos, en relación al punto de equilibrio evaluado, con un déficit que oscilan entre los 25.000 a 60.000 dólares, pero se analiza el valor de la vida humana en estas zonas tan distantes y con condiciones peculiares.

Palabras clave: evaluación financiera; salud pública; costos; Ecuador.

\begin{abstract}
The objective of this study is to analyze the value of financial evaluations as a tool for management decision making in health facilities in areas of difficult access and crystallize the quality of health services through efficiency, effectiveness and effectiveness. The methodology was carried out through the analytical-descriptive study of data focused on eight rural dispensaries of peasant social insurance in the province of Morona Santiago during 2014. The costing of health services was executed by cost centers using Winsig software, the billing of health benefits according to the current tariff and using the equilibrium point equation was determined the financial reality in the territory. It is concluded that the rural health units work at a loss, generating up to three times the expenses, in relation to the assessed balance point, with a deficit ranging between 25,000 and 60,000 dollars, but the value of human life is analyzed in these areas so distant and with peculiar conditions.
\end{abstract}

Keywords: financial evaluation; public health; costs; Ecuador. 


\section{Resumo}

O objetivo deste estudo é analisar o valor das avaliações financeiras como ferramenta para a tomada de decisão gerencial nas unidades de saúde em áreas de difícil acesso e cristalizar a qualidade dos serviços de saúde por meio da eficiência, eficácia e efetividade. A metodologia foi realizada por dados analíticos estudo descritivo focada em oito clínicas rurais na previdência rural na província de Morona Santiago durante 2014. centro de custo que custam os serviços de saúde foi executado por software Winsig, o faturamento dos benefícios de saúde de acordo com a tarifa atual e usando a equação do ponto de equilíbrio foi determinada a realidade financeira no território. Conclui-se que as unidades de saúde rurais trabalhar em um trabalho perda, levando a gastos triplo em relação ao ponto de equilíbrio avaliada, com um déficit que variam de $\$ 25.000$ a $\$ 60.000$, mas o valor da vida humana é analisado em estas áreas tão distantes e com condições peculiares.

Palavras chave: avaliação financeira; saúde pública; custos; Equador.

\section{Introducción}

La región amazónica históricamente alejada de la geografía nacional y con una brecha de necesidades básicas insatisfechas en relación a la costa y serranía del Ecuador, se vio durante décadas en condición abandono en todos los ámbitos, no siendo excepción el área de la salud con un sistema de salud que ejerció poca institucionalidad y presencia en el territorio, dejando esta responsabilidad a terceras personas y tomando protagonismo instituciones ajenas, como: organizaciones no gubernamentales, gobiernos autónomos descentralizados municipales y provinciales y porque no manifestarlo, el Instituto Ecuatoriano de Seguridad Social, que a partir del año 1968 promueve un programa enfocado a garantizar prestaciones de salud a la población vulnerable que radicaba en las zonas rurales y que eran el motor de la producción nacional, mediante la digna labor agrícola, ganadera y pesquera del ecuador, vigente hasta la actualidad, conocido como Seguro Social Campesino (Díaz, 2014).

Las zonas rurales del país y en especial las localizadas en la región amazónica, durante los últimos cinco años y a través de políticas inclusivas que buscan disminuir la brecha de desigualdad, han sido beneficiadas por innumerables esfuerzos de los gobiernos de turno enfocados en mejoramiento de la vialidad, inversión en infraestructura, equipamiento, recursos 
humanos y en el campo de la salud, mediante la construcción de unidades, acorde a las necesidades de cada punto de la geografía, implementando la repotenciación de varias unidades operativas categorizándolas según su capacidad resolutiva y a su vez determinando cada uno de los requisitos y condiciones para su construcción y operatividad; estos planes se han visto afectados por factores como la dispersión de las personas pertenecientes a las nacionalidades indígenas allí establecidas y por la accesibilidad en territorio de muchas poblaciones que aún se mantienen inaccesibles por vía terrestre o en su defecto se encuentran en línea de frontera con vías de comunicación de tercer orden.

La salud en el Ecuador se constituye en un derecho irrenunciable, el mismo debe garantizarse de manera holística para solventar las necesidades del ser humano, fomentándose como política nacional el sistema de salud, así como el acceso libre de discriminación en factores, como: edad, identidad de género, etnia, nivel académico, estatus social o cualquier otra particularidad a nivel personal, familiar o comunitaria (Asamblea Nacional Constituyente de Ecuador, 2008).

En este orden de ideas, el modelo de atención integral de salud familiar, comunitario e intercultural MAIS-FCI, es un conjunto de estrategias, normativas, procedimientos, herramientas y recursos, que articulados de manera armónica buscan satisfacer la demanda de salud de los diversos grupos poblacionales de la sociedad y el medio ambiente circundante, teniendo como base estructurada la integración de los diferentes niveles de salud. Sin duda, el modelo actual se operativiza fomentando la importancia del individuo, familia y comunidad, ubicándolo como eje central de todos los esfuerzos del sistema nacional de salud, otorgando la preponderancia a la vida humana como motor para el funcionamiento de regímenes de salud eficientes con la calidad y calidez, que se requiere en cada uno de los entornos en los que se desempeña el individuo (Ministerio de Salud Pública, 2010).

El primer nivel de atención constituido por puestos y centros de salud constituye la puerta de entrada al sistema nacional de salud, el mismo que ha sido destinado como ente ofertante de salud en las parroquias rurales del ecuador y bajo su responsabilidad está la resolución del 80\% de las afecciones de la población, razón por la cual se fomentó el mejoramiento integral de estos establecimientos con la finalidad de descongestionar los hospitales de segundo y tercer nivel y a su vez institucionalizar la salud en las zonas rurales del país, fortaleciendo los equipos de 
atención integral en salud; se establece los requisitos para el funcionamiento de estas unidades de salud en torno a la población asignada, acorde al Modelo de Atención Integral de Salud, la misma que define que para el funcionamiento de un centro de salud tipo A, que cuenta con la dotación de profesionales de Salud (médico y enfermera), y consecuente a ello el respectivo equipamiento se requiere de más de 10000 habitantes, realidad poco aplicable en la amazonia (Ministerio de Salud Pública, 2010).

El financiamiento de la salud en nuestra realidad está solventado de manera heterogénea por diversos entes generadores de recursos, por una parte en sector público se ve solventado por los diferentes ingresos que genera el fisco en torno a las exportaciones petroleras, no petroleras, impuestos, entre otros; además contribuyen a dichas arcas toda la población laboral en relación de dependencia y los empleadores mismos que solventan la seguridad social y de manera particular privadas el bolsillo de las personas, a través de los seguros privados o personas con capacidad de pago.

Los costos de la salud en establecimientos de salud públicos se cubren mediante los recursos aportados por el ente de financiamiento para promover una determinada prestación de salud, dirigida a la colectividad, dentro de la cual se describen: costos fijos, los mismos que no varían de acuerdo a la cantidad de prestaciones realizadas en contraste con los costos variables; costos directos los mismos que se destinan para la producción del servicio y los costos indirectos que aunque no están destinados directamente a la producción complementan dicho fin, otorgando calidad al servicio ofertado (Pesantez, 2012).

La gestión productiva de las instituciones de salud se basa en procesos sistemáticos enfocados en el análisis la productividad, así como la administración eficiente de los recursos asignados a cada servicio de salud y a su vez la promoción de la calidad e integralidad de los servicios de salud a disposición de la población en cada uno de los niveles de complejidad del régimen de salud del país. Esto surge en la década de los setenta con el plan piloto denominado Sistema de Información Gerencial (SIG), el mismo que en la década de los ochenta se computariza mediante un software aplicado en países de la subregión como Venezuela, no siendo hasta el año 2007, cuando se moderniza dicho software, acorde a la realidad actual y se difunde la metodología para su aplicación de manera global (Organización Panamericana de la Salud, 2010). En el Ecuador la 
gestión en el área de la salud se aplica de manera secuencial en hospitales y establecimientos de gran complejidad, con una escasa o nula aplicación en centros y puestos de salud del primer nivel de atención, solo empleándose en planes pilotos implementados por la Universidad Particular de Loja y el Ministerio de la Salud Pública de la República del Ecuador en el año 2014 (2014).

Basado en lo expuesto anteriormente, la presente Investigación se basó en estudios realizados mediante el pilotaje de evaluaciones financieras de los establecimientos de salud realizados durante el año 2014 en el país, por los resultados obtenidos se planteó como hipótesis que la evaluación financiera de los establecimientos de salud públicos de primer nivel de atención en zonas de la amazonia es útil para la administración de recursos. Como objetivo primario se determinó la aplicabilidad de las evaluaciones financieras de los establecimientos de salud públicos de primer nivel de la amazonia para la administración de recursos. Los objetivos secundarios se centraron en la ejecución del análisis financiero de las unidades de salud rural de la amazonia y en la identificación de los factores que influyen de manera complementaria en la asignación recursos en los establecimientos de salud de la amazonia.

\section{Importancia del problema}

En la actualidad, los establecimientos del primer nivel de atención del país, sin exceptuar la amazonia, son dotados de recursos, basado en consumos históricos con un reajuste anual, evidenciándose la subutilización de metodologías relacionados con la gestión productiva de los servicios de salud recomendada por la Organización Panamericana de la Salud (OPS)(2017), los cuales están destinados a fortalecer la administración, organización y gestión las empresas prestadoras de salud de manera eficiente acorde con los cambios a los que se afrontan los países de la región (Thompson, Gamble, Peteraf y Strickland, 2008).

Los equipos de salud y administradores manejan hipotéticamente idiomas diferentes a la hora de destinar y manejar los recursos dirigidos a la población, muchas veces ocasionadas por el desconocimiento de sistemas eficientes de manejo de recursos, a lo que se suma el desinterés de aplicación de estrategias que propiciarían una atención de calidad en los servicios de salud.

La evaluación financiera y su uso rutinario para la asignación de recursos se queda corta ante la complejidad de la salud y el valor de los modelos de atención en salud los mismos que promulgan el valor de la vida humana como eje central del accionar de los servicios de salud por 
lo que resulta inherente la valorar factores adicionales que complementan y justifican la toma de decisiones por parte de los gerentes y administradores de recursos (Vargas, 2005).

\section{Metodología}

Se aplicó el tipo de estudio analítico-descriptivo, de corte transversal para recabar datos. El universo estuvo constituido por 16 unidades de salud, pertenecientes al régimen especial de Seguro Social Campesino de la provincia de Morona Santiago, las cuales se disgregaron por criterios generales de accesibilidad, siendo evaluadas las 8 unidades de salud más distantes. Se recolectó la información de costos, a partir de datos de consolidados mensuales de producción, formatos de requisición de medicamentos insumos y biomateriales, roles de pago de los servidores y acta de activos fijos, datos que fueron consolidados en un formulario prediseñado para este propósito e ingresadas por centro de costo en el programa Winsig. Los datos de facturación se tomaron en cuanto, en número de prestaciones, relacionándolas con el tarifario vigente tomando en cuenta cuatro prestaciones (consulta médica, consulta odontología, actividades de promoción y visitas domiciliarias). El punto de equilibrio se realizó a través de fórmulas matemáticas y contables con el análisis respectivo de los condicionantes propios y externalidades que complejizaban dicho estudio.

\section{Resultados}

De acuerdo al proceso de costeo de los establecimientos de salud por centros de costo se evidenció que los servicios de salud de la zona rural amazónica, demandan dotación de recursos que oscilan desde los 50.000 a 75.000 dólares anuales para su mantenimiento de manera global, teniendo como mayor disposición de recursos la dotación de recursos humanos y bonificaciones para su permanencia en estos lugares recónditos de la geografía. La consulta médica requiere cerca del $40 \%$ de dicho presupuesto, debido a que es considerado el servicio estrella sobre el cual recaen muchos costos directos relacionados con su oferta. 


\section{Tabla 1}

Costos de los Servicios de Salud

\begin{tabular}{|l|c|c|c|c|c|c|c|c|}
\hline \multicolumn{1}{|c|}{$\begin{array}{c}\text { Costos de } \\
\text { Servicios }\end{array}$} & $\begin{array}{c}\text { Tayu } \\
\mathbf{z a}\end{array}$ & $\begin{array}{c}\text { La } \\
\text { Unión } \\
\text { III }\end{array}$ & $\begin{array}{c}\text { Camba- } \\
\text { naca }\end{array}$ & $\begin{array}{c}\text { Indan } \\
\text { za }\end{array}$ & $\begin{array}{c}\text { El } \\
\text { Rosar } \\
\text { io 1 }\end{array}$ & $\begin{array}{c}\text { Unión } \\
\text { De } \\
\text { Kala- } \\
\text { glas }\end{array}$ & $\begin{array}{c}\text { Bomb } \\
\text { oiza }\end{array}$ & $\begin{array}{c}\text { Rosari } \\
\mathbf{0} 4\end{array}$ \\
\hline Consulta Externa & 18516,35 & 15528,20 & 29008,05 & 30941,83 & 22832,39 & 21798,51 & 29756,54 & 25481,45 \\
\hline Visitas & 5610,28 & 8821,48 & 6662,82 & 5704,48 & 5612,28 & 7312,23 & 6547,00 & 5433,96 \\
Domiciliarias & & & & & & & & \\
\hline Odontología & 8717,92 & 4257,64 & 4265,20 & 4137,88 & 4544,32 & 8445,76 & 11193,92 & 14555,62 \\
\hline Actividades De & 6151,08 & 4949,52 & 9154,88 & 6151,08 & 7652,98 & 8697,36 & 6151,08 & 6151,08 \\
\hline Promoción & & & & & & & & \\
\hline Administración & 15728,33 & 16979,49 & 25756,49 & 17701,45 & 16829,43 & 22102,43 & 17462,96 & 21014,07 \\
& & & & & & & & \\
\hline
\end{tabular}

Fuente: Elaboración propia. Cuadros Gerenciales Winsig $N^{\circ} 1$ y 4.

Mediante el proceso de valoración de costos por atención para tener un parámetro manejable por la colectividad, se denotó que las prestaciones de salud ofertadas por estos establecimientos por atención médica en consulta externa cuestan más de 20 dólares, consulta odontológica cuesta aproximadas a los 10 dólares, las actividades de promoción cuestan cerca de los 200 dólares y las visitas domiciliarias más de 75 dólares.

\section{Tabla 2}

Costos de la Atención por Prestaciones.

\begin{tabular}{|l|l|r|r|r|}
\hline \multicolumn{1}{|c|}{ Dispensarios } & \multicolumn{1}{|c|}{ Servicio } & $\begin{array}{c}\text { Costo } \\
\text { total } \\
\text { anual }\end{array}$ & $\begin{array}{c}\text { Número } \\
\text { de } \\
\text { atencione } \\
\text { s }\end{array}$ & $\begin{array}{c}\text { Costo } \\
\text { promedio } \\
\text { por } \\
\text { atención }\end{array}$ \\
\hline \multirow{2}{*}{ Tayuza } & Consulta externa & 18516,3 & 783 & 23,65 \\
& Visitas domiciliarias & 5610,28 & 70 & 80,15 \\
\cline { 2 - 6 } & Odontología & 8717,92 & 649 & 13,43 \\
\hline
\end{tabular}




\begin{tabular}{|c|c|c|c|c|}
\hline & $\begin{array}{l}\text { Actividades de } \\
\text { promoción }\end{array}$ & 6151,08 & 23 & 267,44 \\
\hline \multirow[t]{4}{*}{ La Unión III } & Consulta externa & 15528,2 & 895 & 17,35 \\
\hline & Visitas domiciliarias & 8821,48 & 99 & 89,11 \\
\hline & Odontología & 4257,64 & 334 & 12,75 \\
\hline & $\begin{array}{l}\text { Actividades de } \\
\text { promoción }\end{array}$ & 4949,52 & 37 & 133,77 \\
\hline \multirow[t]{4}{*}{ Cambanaca } & Consulta externa & 30941,8 & 1725 & 17,94 \\
\hline & Visitas domiciliarias & 5704,48 & 71 & 80,34 \\
\hline & Odontología & 4137,88 & 634 & 6,53 \\
\hline & $\begin{array}{l}\text { Actividades de } \\
\text { promoción }\end{array}$ & 6151,08 & 25 & 246,04 \\
\hline \multirow[t]{4}{*}{ Indanza } & Consulta externa & 30941,8 & 1898 & 16,30 \\
\hline & Visitas domiciliarias & 5704,48 & 73 & 78,14 \\
\hline & Odontología & 4137,88 & 739 & 5,60 \\
\hline & $\begin{array}{l}\text { Actividades de } \\
\text { promoción }\end{array}$ & 6151,08 & 25 & 246,04 \\
\hline \multirow[t]{4}{*}{ Rosario 1} & Consulta externa & 22832,3 & 1641 & 13,91 \\
\hline & Visitas domiciliarias & 5612,28 & 94 & 59,71 \\
\hline & Odontología & 4544,32 & 814 & 5,58 \\
\hline & $\begin{array}{l}\text { Actividades de } \\
\text { promoción }\end{array}$ & 7652,98 & 27 & 283,44 \\
\hline \multirow{5}{*}{$\begin{array}{l}\text { Unión de } \\
\text { Kalaglas }\end{array}$} & Consulta externa & 21798,5 & 1216 & 17,93 \\
\hline & & 1 & & \\
\hline & Visitas domiciliarias & 7312,23 & 75 & 97,50 \\
\hline & Odontología & 8445,76 & 783 & 10,79 \\
\hline & $\begin{array}{l}\text { Actividades de } \\
\text { promoción }\end{array}$ & 8697,36 & 26 & 334,51 \\
\hline Bomboiza & Consulta externa & 29756,5 & 1663 & 17,89 \\
\hline
\end{tabular}




\begin{tabular}{|l|l|r|r|r|}
\hline & Visitas domiciliarias & 6547,00 & 78 & 83,94 \\
\cline { 2 - 5 } & Odontología & 11193,9 & 589 & 19,00 \\
\hline Rosario 4 & $\begin{array}{l}\text { Actividades de } \\
\text { promoción }\end{array}$ & 6151,08 & 25 & 246,04 \\
\cline { 2 - 5 } & Consulta externa & 25481,4 & 1117 & 22,81 \\
\cline { 2 - 5 } & Visitas domiciliarias & 5433,96 & 82 & 66,27 \\
\cline { 2 - 5 } & $\begin{array}{l}\text { Odontología } \\
\text { Octividades de }\end{array}$ & 6151,08 & 1220 & 11,93 \\
& $\begin{array}{l}\text { Promoción } \\
\text { Action }\end{array}$ & 26 & 236,58 \\
\hline
\end{tabular}

Fuente: Elaboración propia. Cuadros Gerenciales Winsig No 1 y 4.

A través de la facturación o planillaje de la productividad, como herramienta el tarifario nacional de prestaciones de salud vigente, se pudo dilucidar que dichos servicios de salud a su vez facturan valores totales anuales que van desde los 32.000 dólares anuales, reflejando que la consulta externa, por ser también la prestación más demanda, es la que genera mayores ingresos en los establecimientos de salud de primer nivel en la amazonia ecuatoriana (Ministerio de Salud Pública de la República del Ecuador (2014).

Tabla 3

Planillaje de los servicios de salud.

\begin{tabular}{|l|l|r|}
\hline \multicolumn{1}{|c|}{ Dispensarios } & \multicolumn{1}{c|}{ Servicio } & \multicolumn{1}{c|}{$\begin{array}{c}\text { Planillaje total } \\
\text { anual }\end{array}$} \\
\hline \multirow{5}{*}{ TAYUZA } & Consulta externa & 12773,17 \\
\cline { 2 - 3 } & Visitas domiciliarias & 1495,90 \\
\cline { 2 - 3 } & $\begin{array}{l}\text { Actividades de } \\
\text { promoción }\end{array}$ & 272,55 \\
\cline { 2 - 3 } & Odontología & 5585,40 \\
\hline \multirow{2}{*}{ LA UNION III } & Consulta externa & 13604,00 \\
\cline { 2 - 3 } & Visitas domiciliarias & 2115,63 \\
\hline
\end{tabular}




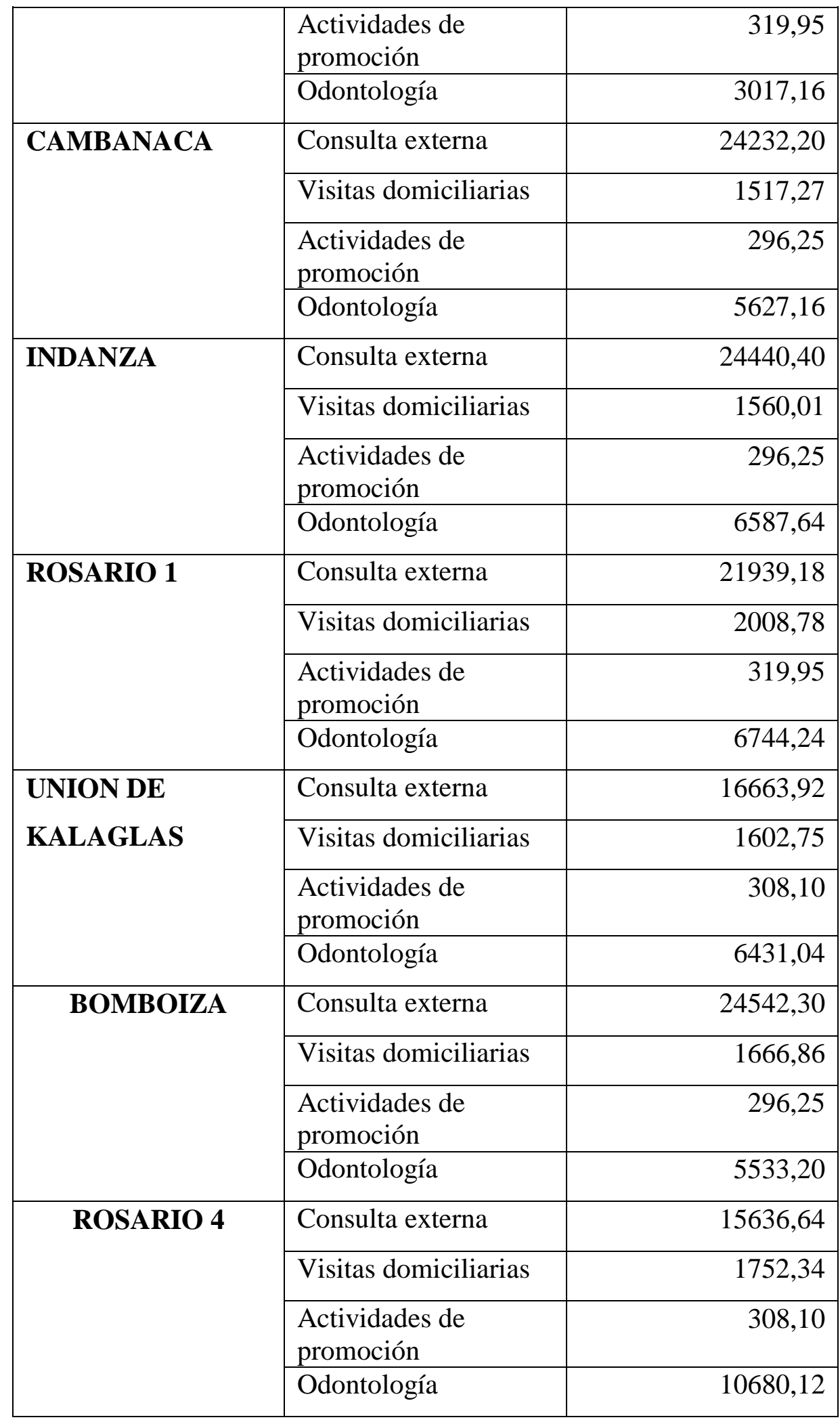

Fuente: Elaboración propia. Matrices de Facturación prediseñadas. 
Como punto esencial de la evaluación financiera se procedió al cálculo del punto de equilibrio para lo cual se empleó una formula contable correspondiente observándose que los establecimientos de salud de primer nivel de la amazonia trabajan a perdidas generando déficits monetarios que van desde los 50.000 a 75.000 dólares anuales lo que pone en la palestra la dotación de recursos superávit que existe para el mantenimiento de estos servicios en relación a los ingresos que genera.

\section{Imagen1}

Fórmula para el cálculo del punto de equilibrio.

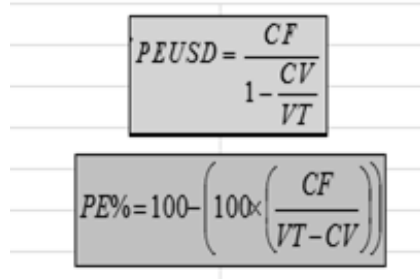

Nota: (PEUSD representa el punto de equilibrio en dólares, PE\% representa el punto de equilibrio en porcentaje, $\mathrm{CF}$ representa el costo fijo, $\mathrm{CV}$ representa el costo variable y VT representa el total de ventas o facturación)

Fuente: Thompson et al., (2008)

Tabla 4

Cálculo del Punto de equilibrio.

\begin{tabular}{|c|r|c|c|r|r|r|r|c|}
\hline $\begin{array}{c}\text { Parámetr } \\
\text { os }\end{array}$ & Tayuza & $\begin{array}{c}\text { La } \\
\text { unión } \\
\text { III }\end{array}$ & $\begin{array}{c}\text { Cambana } \\
\text { ca }\end{array}$ & $\begin{array}{c}\text { Indanz } \\
\text { a }\end{array}$ & $\begin{array}{c}\text { El } \\
\text { rosario } \\
\mathbf{1}\end{array}$ & $\begin{array}{c}\text { Unión } \\
\text { de } \\
\text { kalagla } \\
\text { s }\end{array}$ & $\begin{array}{c}\text { Bombo } \\
\text { iza }\end{array}$ & $\begin{array}{c}\text { Rosa } \\
\text { rio 4 }\end{array}$ \\
\hline Costos & 44229 & 4355 & 64353,28 & 54233 & 46976 & 57877 & 60657 & 6214 \\
Fijos &, 80 & 0,52 & &, 16 &, 70 &, 69 &, 34 & 9,17 \\
\hline Costos & 10494 & 6985 & 10494,16 & 10403 & 10494 & 10478 & 10454 & 1048 \\
Variables &, 16 &, 81 & &, 56 &, 70 &, 60 &, 16 & 7,01 \\
\hline Total & 54723 & 5053 & 74847,44 & 64636 & 57471 & 68356 & 71111 & 7263 \\
\hline
\end{tabular}




\begin{tabular}{|c|r|r|l|r|r|r|r|r|}
\hline Costos &, 96 & 6,33 & &, 72 &, 40 &, 29 &, 50 & 6,18 \\
\hline Ingresos & 20127 & 1905 & 31672,88 & 32884 & 31012 & 25005 & 32038 & 2837 \\
Tarifados &, 02 & 6,74 & &, 30 &, 15 &, 81 &, 61 & 7,20 \\
\hline Punto De & 92414 & 6875 & 96240,65 & 79331 & 71005 & 99625 & 90035 & 9858 \\
Equilibrio &, 31 & 4,51 & &, 00 &, 34 &, 36 &, 97 & 0,25 \\
\hline \% Punto & - & - & $-203,86$ & - & - & - & - & - \\
De & 359,1 & 260, & & 141,2 & 128,9 & 298,4 & 181,0 & 247, \\
Equilibrio & 6 & 79 & & 4 & 6 & 1 & 2 & 39 \\
\hline
\end{tabular}

Fuente: Elaboración propia Matrices de Facturación prediseñadas y Cuadros Gerenciales Winsig $\mathrm{N}^{\mathrm{o}} 1$ y4.

\section{Discusión}

La multidimensionalidad de la salud crea una ambiente único para gerente en torno a la toma de decisiones para la dotación de recursos en los establecimientos de salud siendo aún más complejo cuando se analizan las características particulares de la amazonia, tales como la dispersión poblacional, la ruralidad, la accesibilidad y como parte importante la desinterés del talento humano por laborar en sitios tan alejados, todos ellos se conjugan y dificultan las decisiones del gerente que muchas veces no basta con un simple y frio análisis de números sino que sopesa el valor humano y los esfuerzos desmesurados con la única finalidad de garantizar la salud y la vida a este grupo poblacional tan desfavorecido de nuestra patria. De ahí surge el interrogante a cerca de los factores externos que condicionan las decisiones gerenciales en salud como complemento a la evaluación financiera en estos establecimientos con todas sus particularidades.

\section{Conclusiones}

Las evaluaciones financieras enfocadas en los establecimientos de salud son una herramienta de gran utilidad, en torno a la toma de decisiones para la administración de todo tipo de recursos por parte de los entes que realizan el gerenciamientos de las mismas, por lo que resultó es de gran utilidad, al evaluar el caso particular de los ocho dispensarios rurales del Seguro Social Campesino de la provincia de Morona Santiago, evidenciándose que dichos establecimientos generaban una pérdida de recursos que en ocasiones triplicaba su productividad en relación al punto de equilibrio evaluado. 
Factores externos como el aspecto demográfico, geográfico, vial, servicios básicos en las localidades, permanencia del profesional, pero sobre todo la premisa de la salud como un derecho, influyen en los gerentes a la hora de distribuir los recursos ya que aunque estos establecimientos generen pérdidas cuantiosas se requiere de su permanencia y operatividad no solo para generar prestaciones de salud sino para garantizar la vida de las personas pertenecientes en la mayor parte de los casos a nacionalidades indígenas dispersas en la inmensidad de la amazonia ecuatoriana.

El valor de la evaluación financiera de los establecimientos de salud de primer nivel basados en la APS, va destinado a la formulación de estrategias que fomenten el fortalecimiento de los equipos básicos de salud no solo mediante la implementación de indicadores de productividad sino más bien deben enfocarse en la aplicación indicadores de gestión comunitaria acordes a la normativa en salud vigente, tales como ejecución de diagnósticos comunitarios dinámicos, planes de salud, conformación de grupos organizados para garantizar la participación comunitaria, programación y monitoreo de actividades, implementación de salas situacionales, entre otras, instrumentos con los que el cliente externo generaría un valor agregado a su labor asistencial.

\section{Referencias Bibliográficas}

Asamblea Nacional Constituyente de Ecuador. (2008). Constitución de la República del Ecuador. Registro oficial 449.

Díaz, Z. (2014). Evaluación financiera de los dispensarios del Seguro Social Campesino de Bomboiza, Cambanaca, Indanza, Rosario I, Rosario IV, Tayuza, Unión III y Unión de Kalaglas, pertenecientes a los distritos 14d04 y 14d06. Morona Santiago. Universidad Técnica Particular de Loja. 2016.

Ministerio de Salud Pública. (2010). Lineamientos Operativos del Modelo de Atención Integral en Salud y de la Red pública integral de salud. Primera Edición. Quito: MSP.

Ministerio de Salud Pública de la República del Ecuador. (2014). Tarifario de Prestaciones del Sistema Nacional de Salud. Segunda Edición. Quito. 
Organización Panamericana de la Salud. (2010). Metodología de gestión productiva de los servicios de salud. Washington, DC: Organización Panamericana de la Salud.

Organización Panamericana de la Salud. (2007). La Renovación de la Atención Primaria en salud en las Américas. Washington, DC: Organización Panamericana de la Salud.

Pesantez, M. (2012). Guía Didáctica de Economía y Salud. Loja. Universidad Técnica Particular de Loja.

Thompson, G; Gambel, J; Peteraf, M \& Strickland, A. (2008). Administración Estratégica, teoría y casos. Decimoctava Edición. Las Vegas: Mc Graw-Hill interamericana.

Vargas, J. (2005). Planificación en los Servicios de Salud. Universidad de Costa Rica. San José. Costa Rica. 